Sutherland, E. W. (1955). Polysaccharide phosphorylase, liver. In Methods in Enzymology, Vol. I, p. 215. Ed. by S. P. Colowick and N. O. Kaplan. Academic Press, New York.

M. LUdwiG, S. WOLFSON, and O. RENNERT The Department of Pediatrics, University of Florida College of Medicine, Gainesville, Florida 32601, U.S.A.

*Correspondence to Dr. O. Rennert.

\section{Direct Current Cardioversion in a Case of Congenital Atrial Flutter}

The congenital type of atrial flutter has a poor prognosis when unresponsive to digoxin (Moller, Davachi, and Anderson, 1969). Recorded experience with cardioversion is limited but it has been suggested that this type of flutter is refractory to electric countershock (Rodriguez-Coronel, Sueblingvong, and Hastreiter, 1968).

We report an infant with congenital heart defects and congenital atrial flutter who appeared resistant to digoxin and $\beta$-blockade (practolol) but readily reverted to sinus rhythm with direct current (DC) cardioversion.

\section{Case Report}

This was the second pregnancy of a 25-year-old whose health was good until mild hypertension developed at term. Labour was induced at $\mathbf{4 0}$ weeks, and the fetal heart was noted to be rapid and irregular. A mature female infant weighing $3.8 \mathrm{~kg}$ was delivered by emergency caesarean section. No meconium staining was evident, and the Apgar score at one minute was 9. The infant remained active, sucked well, and was pink breathing air.

The apex beat was consistently $180-200 /$ minute. No cardiac murmurs were heard at this time and there were no signs of respiratory distress. $X$-ray showed slight cardiomegaly and clear lung fields. ECG showed atrial flutter with varying AV block and a ventricular rate around $180 /$ minute.

An attempt was made to control the ventricular rate using digoxin orally. A total dose of $0.3 \mathrm{mg}$ over the first 24 hours was given, and $0.075 \mathrm{mg}$ daily thereafter. After 3 days there was no change in the underlying arrhytnmia or the ventricular rate (Fig.). At this time a soft systolic murmur became audible at the left sternal edge.

At the age of 4 days cardiac catheterization revealed an atrial septal defect with a pulmonary-systemic flow ratio of $2: 1$ and a minor degree of aortic coarctation. During the procedure the atrial flutter reverted to sinus rhythm for two short periods.

At 5 days digoxin was stopped. After 24 hours, and with continuous ECG monitoring, practolol was given twice within one hour by slow i.v. injection in a dose of $0.5 \mathrm{mg}$ diluted in $10 \mathrm{ml}$ saline. No effect was noted on the heart rate or rhythm. Oral practolol $2.2 \mathrm{mg} / \mathrm{kg}$ twice daily was given and increased to four times daily without effect on rate or rhythm (Fig.).

By 10 days the baby's condition was deteriorating with tachypnoea and feeding difficulty. The liver was palpable two fingers' breadth below the costal margin. $X$-ray revealed increasing cardiomegaly. DC cardioversion was, therefore, attempted.

No anaesthetic was given. Adult electrodes $(9 \mathrm{~cm}$ diameter) were placed on the right midsternal border and on the apex with about nine-tenths of the surface area of the paddles masked by insulating tape. A shock of 10 watt-seconds was given from a Lown cardiorater. There was immediate reversion to sinus rhythm with an
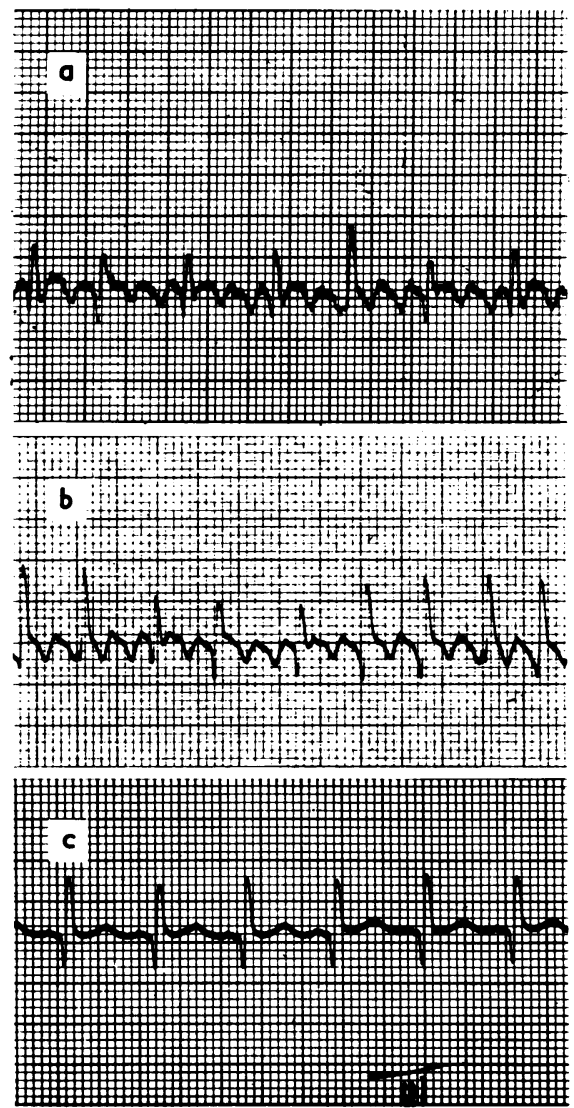

FIG.-Electrocardiogram (lead III) in congenital atrial flutter: effects of treatment; (a) on digoxin, (b) on practolol, and $(c)$ after cardioversion. 
initial heart rate of 160 /minute, which slowed to 140 / minute within a few days. Practolol $8 \mathrm{mg}$ four times daily by mouth was continued.

At 4 months of age the heart remained in sinus rhythm at 140/minute and ECG showed more right ventricular hypertrophy than expected at this age. On $x$-ray slight cardiomegaly persisted. The baby is well.

\section{Discussion}

Congenital atrial flutter is usually recognized before birth or within the course of the first week. It is continuous, whereas flutter occurring after the first few weeks may be paroxysmal.

Rodriguez-Coronel et al. (1968) suggest that congenital atrial flutter is refractory to treatment both by digoxin and DC shock but is well tolerated. Moller et al., however, showed that this early type is often responsive to digoxin (13 out of 24 cases) but those who were not responsive to digoxin had a poor prognosis (5 out of 11 cases died). Coexistent congenital heart defects worsen the prognosis.

In our case, digoxin failed to induce sinus rhythm or slow the ventricular response but it is possible that larger dosage would have been more effective.

Practolol is a cardioselective $\beta$-adrenergic blocking agent which has proved useful in supraventricular tachycardias in adults (British Medical Journal, 1970). Its value in infancy has not been documented, but in this case there was no effect with i.v. or prolonged oral administration.

Electric cardioversion has been used successfully in a newborn with atrial flutter (Hassenrück, Chojnacki, and Barker, 1965), but there was no response in the case of congenital flutter reported by Rodriguez-Coronel et al. The technique and amount of electrical energy may be critical factors in obtaining a response (Treister and Kleiger, 1969). In our case a single shock of low energy was effective and the procedure appeared simple and safe. Only transient erythema of the skin occurred over the paddle sites. There is a hazard of postcardioversion arrhythmia when the procedure is carried out in the digitalized patient and this drug should be stopped at least 24 hours before elective cardioversion (Lown, 1967). We had stopped digoxin one week before attempting cardioversion.

Digoxin has generally been the treatment of first choice in infantile flutter, but in cases that do not respond our experience suggests that there should be no hesitation in a trial of electric cardioversion. The time lapse involved in an unsuccessful trial of digoxin suggests that cardioversion should be considered the treatment of first choice when the arrhythmia is poorly tolerated, particularly where there is associated cardiac malformation which worsens the prognosis.

\section{Summary}

A case of congenital atrial flutter reverted to sinus rhythm with a single DC shock of 10 watt-seconds, after apparent failure of digoxin and practolol.

We are grateful to Dr. M. B. Mathews who advised throughout on management, and to Drs. D. G. Julian and $\mathrm{H}$. H. Bain who undertook the cardiac catheterization.

\section{REFERENCES}

British Medical fournal (1970). Leading article. Beta blockade in flutter, 4, 636

Hassenrück, A., Chojnacki, B., and Barker, H. J. (1965). Cardioversion of auricular flutter in a newborn infant. American Fournal of Cardiology, 15, 726.

Lown, B., (1967). Electrical reversion of cardiac arrhythmias. British Heart Fournal, 29, 469.

Moller, J. H., Davachi, F., and Anderson, R. C. (1969). Atrial flutter in infancy. Fournal of Pediatrics, 75, 643.

Rodriguez-Coronel, A., Sueblingvong, V., and Hastreiter, A. R. (1968). Clinical forms of atrial flutter in infancy. Fournal of Pediatrics, 73, 69.

Treister, B., and Kleiger, R. E. (1969). Atrial flutter. (Letter.) fournal of Pediatrics, 74, 1005.

R. P. C. BARCLAY and D. G. D. BARR

Medical Paediatric Unit, Western General Hospital, Edinburgh EH4 $2 X U$.

^Correspondence to Dr. D. G. D. Barr. 